\title{
A synthesized chart for scheduling of production systems with automated guided vehicle
}

\author{
Hideaki Hase and Norio Okino \\ School of Engineering, The University of Shiga Prefecture \\ Hikone, Shiga 522, JAPAN \\ Tel: +81-749-28-8394 Fax: +81-749-28-8597 \\ E-mail: hase@mech.usp.ac.jp
}

\begin{abstract}
In this paper, an effective use of Geometric Gantt Chart (GGC) is proposed where GGC is a synthesized chart of Gantt chart on job-shop process and operation diagram on Automated Guided Vehicle (AGV) which was proposed by one of the authors. The method proposed here is: (1) planning successively the loci of the vehicles and the jobs, (2) evaluating and improving the carriage efficiency of the vehicles and (3) repeating these process. It is confirmed that the proposed method is effective for production systems with AGV to make the time required shorter than the conventional ways.
\end{abstract}

\section{Keywords}

Production Scheduling, Automated Guided Vehicle (AGV), Gantt Chart, Geometric Gantt Chart (GGC)

\section{INTRODUCTION}

Lately, Automated Guided Vehicle (AGV) systems have come into use in many production factories to transport parts and products. Requirements of products became so various that job-shop process got introduced into factories more and more. Job-shop process is virtually unable to be managed without AGV system.

A lot of researches on production scheduling have been made (Adelsberger \& Conen 1995; Kurbel \& Ruppel 1995) and their outcome is applied also in the factories using AGV. These researches, however, rarely consider the transportation of AGV system. Though there are a lot of researches on operation scheduling for AGV itself (Błazewicz, et al. 1993; Kwok \& Norrie 1992), most of them set their problem with random calls. 
On the scenes of AGV operation in factories, its scheduling is quite a difficult problem and becomes more and more important as pervading of AGV systems. Nowadays in most factories, AGV is operated in a simple way, $i$. e., they are triggered by the calls from jobs which have finished there tasks on machines. In this case, on condition that moving time of AGV is not much less than task time, the jobs spend much idle time because of the lack of insight and of unnecessary waits. Such waste is caused by temporal impassability on singletruck, by capacity of vehicle itself, by constraint due to accompanying loads and so on. The latter two rely on the number of vehicles.

On the other hand, Gantt chart is utilized for production scheduling. This chart that has machine axis and time axis aids intuitive comprehension of schedule on jobs and insight into improvements. Gantt chart is sometimes extended to include notations of various constraints to exhibit the advantages. It is expected that to express constraints due to AGV system in this kind of chart is effective for scheduling of production systems with AGV. However, there was not any precedent proposal for such chart probably because the constraints were too complex to write down with the scheduling chart.

One of the authors showed an idea of a notation to do this called Geometric Gantt Chart (GGC), i. e., a chart which consists of a simple map of the plant and time axis which are orthogonal (Okino 1995). It was the first stage. In this paper, as the second stage, an effective utilization of GGC and its effectiveness are discussed. The following two subjects are contained:

- effective use of the scheduling by GGC,

- verification of the effectiveness of the scheduling by GGC.

\section{GEOMETRIC GANTT CHART (GGC)}

\subsection{The Idea of GGC}

GGC is made by setting up time axes to show work time schedule of every machine on the geometrical map of a plant, perpendicular to positions where machines are located respectively. AGV's scheduling panels are also stood up on the guide way along the time axes (Okino 1995). Figure 1 illustrates GGC for an example of a simple plant with 10 machines and 3 vehicles. Schedule bars along the time axes and driving graphs on AGV's schedule panels show a mixed schedule for all jobs, machines and vehicles.

As an example in Figure 1, schedules on machines of job $\mathrm{P}$ are expressed on time axes passing machines $\mathrm{A}, \mathrm{B}$ and $\mathrm{C}$ as task $\mathrm{P}_{i}(i=1,2, \cdots) . t_{s p i X}$ and $t_{f p i X}$ means the starting time and the finishing time of task $i$ of job $\mathrm{P}$ on machine X, respectively. The solid line in Figure 1 is the locus of AGV 1 and the broken line is that of job $P$. The duplicated segment of them from machine A to machine B represents that AGV 1 is transporting the load of job P. Any 


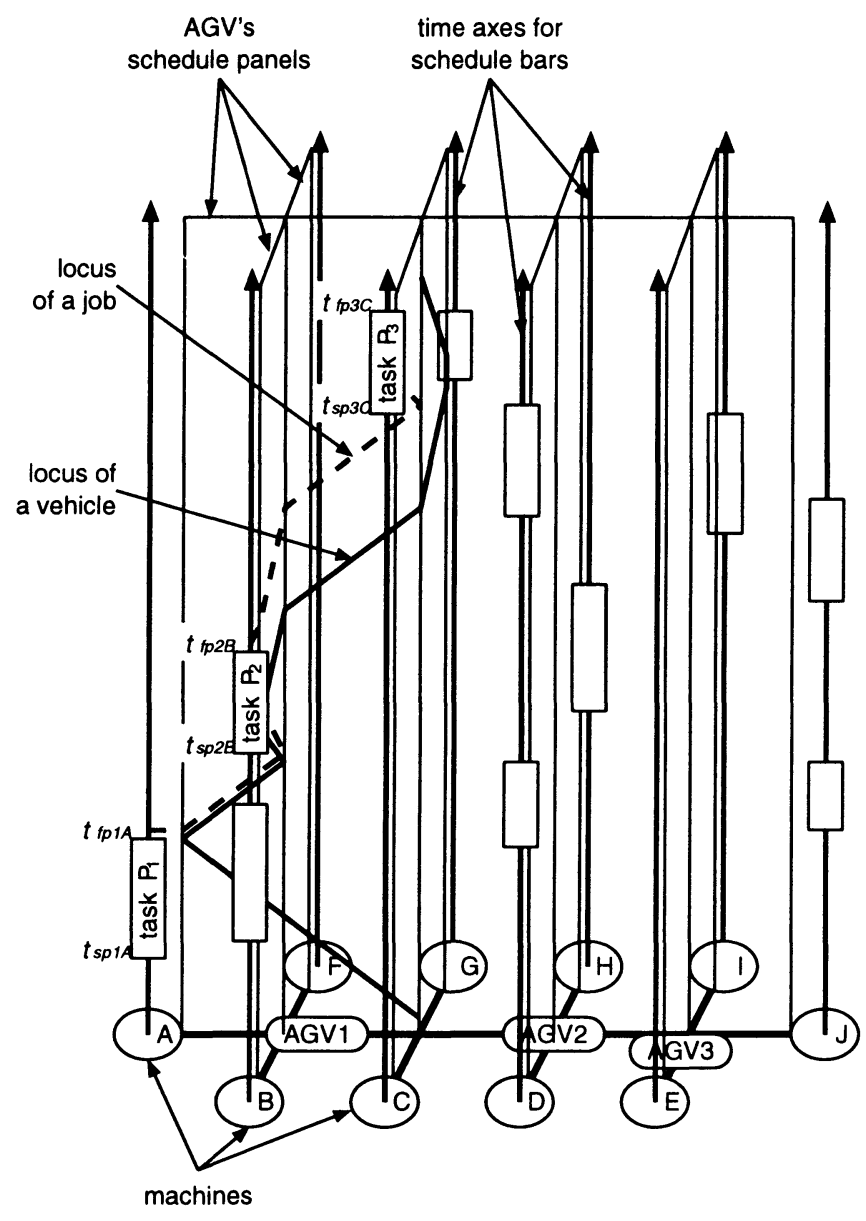

Figure 1 An Example of Geometrical Gantt Chart (GGC)

locus of a job between machines must accompany a vehicle. Since a crossing of the loci of two vehicles means a collision of them, it has to be evaded.

\subsection{An Effective Scheduling by GGC}

A human scheduler coordinates GGC with the geometric map of the plant and time axes. He sets every job and every vehicle at the initial time of the map. Then he makes a temporal plan for each vehicle when to carry its job and which job to carry to its destination. He also writes down expenditures of time of the jobs at work or at wait at their destinations. 
On scheduling, the human scheduler will encounter many alternatives. It is essential to choose the appropriate alternative so that the schedule becomes close to the optimal solution. Accumulation of scheduling by GGC gave some experimental knowledge on effective course of the choices:

- Make carriage efficiency of AGV as high as possible.

- Choose the job which has more work time remained including ideal carriage time, $i$. e., whose task will be finished earlier than the other jobs when chosen. But the former course has the priority.

Ideal here means that it is assumed that no other job exists.

The former course is more significant. The scheduler can estimate the local carriage efficiency from extra time such as terms of emptiness of the vehicle, of stoppage and of escape from others. It improves the temporal plan to reschedule so as to increase its carriage efficiency on or after scheduling.

\section{EFFECTIVENESS OF GEOMETRIC GANTT CHART}

In this section, the effectiveness of GGC for scheduling is verified by means of comparing it to the conventional ways. The comparison is made by applying them to some concrete job-shop scheduling problems with AGV transportation.

\subsection{Examples of Scheduling Problems for Comparison}

It is necessary to show examples in this paper before verifying its effectiveness. The examples here are some requests of 5 jobs to be proceeded on 5 machines linked to each other with AGV guide ways. They are shown in Table 1. Two patterns of guide network as in Figure 2 are taken up: one has a linear trunk and the other a loop.

Each system has 2 vehicles. The vehicles need 1 unit time to pass every link of the guide network. In all of the examples, at the initial time all vehicles are at the pool node and all loads of jobs are at the input node. Each job must come to output node after finishing all of its tasks.

The following constraints are assumed:

- All links are single-truck. No vehicle is able to pass another vehicle.

- No vehicle is allowed to be in the place where other vehicles can reach in 1 unit time.

- The vehicles are allowed either to stop or to turn back only at nodes.

- Each vehicle has a capacity of only 1 job load.

- Each machine is able to stock as many as it is required. 
Table 1 Examples of Job Table

\begin{tabular}{ccccccc}
$\begin{array}{c}\text { request } \\
\text { No. }\end{array}$ & job & \multicolumn{5}{c}{ machine/work time } \\
& & task 1 & task 2 & task 3 & task 4 & task 5 \\
\hline & $\mathrm{P}$ & $\mathrm{A} / 2$ & $\mathrm{~B} / 7$ & $\mathrm{C} / 8$ & $\mathrm{D} / 7$ & $\mathrm{E} / 2$ \\
& $\mathrm{Q}$ & $\mathrm{B} / 6$ & $\mathrm{D} / 2$ & $\mathrm{E} / 7$ & $\mathrm{C} / 7$ & $\mathrm{~A} / 3$ \\
& $\mathrm{R}$ & $\mathrm{E} / 8$ & $\mathrm{C} / 6$ & $\mathrm{~A} / 7$ & $\mathrm{D} / 3$ & $\mathrm{~B} / 2$ \\
& $\mathrm{~S}$ & $\mathrm{D} / 8$ & $\mathrm{~B} / 3$ & $\mathrm{~A} / 6$ & $\mathrm{C} / 2$ & $\mathrm{E} / 6$ \\
& $\mathrm{~T}$ & $\mathrm{C} / 3$ & $\mathrm{~B} / 8$ & $\mathrm{~A} / 8$ & $\mathrm{D} / 6$ & $\mathrm{E} / 3$ \\
\hline & $\mathrm{P}$ & $\mathrm{A} / 3$ & $\mathrm{~B} / 4$ & $\mathrm{C} / 6$ & $\mathrm{D} / 5$ & $\mathrm{E} / 3$ \\
& $\mathrm{Q}$ & $\mathrm{D} / 4$ & $\mathrm{C} / 7$ & $\mathrm{~A} / 7$ & $\mathrm{E} / 5$ & $\mathrm{~B} / 3$ \\
2 & $\mathrm{R}$ & $\mathrm{E} / 5$ & $\mathrm{~A} / 5$ & $\mathrm{~B} / 7$ & $\mathrm{D} / 5$ & $\mathrm{C} / 6$ \\
& $\mathrm{~S}$ & $\mathrm{C} / 6$ & $\mathrm{~A} / 8$ & $\mathrm{D} / 5$ & $\mathrm{~B} / 7$ & $\mathrm{E} / 4$ \\
& $\mathrm{~T}$ & $\mathrm{D} / 8$ & $\mathrm{C} / 4$ & $\mathrm{~A} / 7$ & $\mathrm{~B} / 7$ & $\mathrm{E} / 6$ \\
\hline & $\mathrm{P}$ & $\mathrm{A} / 7$ & $\mathrm{~B} / 5$ & $\mathrm{C} / 5$ & $\mathrm{D} / 4$ & $\mathrm{E} / 8$ \\
& $\mathrm{Q}$ & $\mathrm{D} / 4$ & $\mathrm{~A} / 5$ & $\mathrm{C} / 7$ & $\mathrm{~B} / 8$ & $\mathrm{E} / 6$ \\
3 & $\mathrm{R}$ & $\mathrm{E} / 3$ & $\mathrm{C} / 3$ & $\mathrm{~B} / 5$ & $\mathrm{D} / 6$ & $\mathrm{~A} / 7$ \\
& $\mathrm{~S}$ & $\mathrm{E} / 5$ & $\mathrm{D} / 7$ & $\mathrm{C} / 6$ & $\mathrm{~B} / 5$ & $\mathrm{~A} / 3$ \\
& $\mathrm{~T}$ & $\mathrm{D} / 3$ & $\mathrm{E} / 5$ & $\mathrm{C} / 6$ & $\mathrm{~A} / 5$ & $\mathrm{~B} / 4$ \\
\hline
\end{tabular}

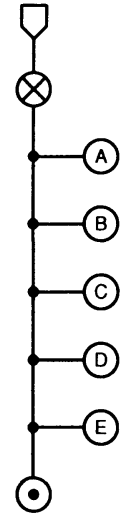

pattern 1

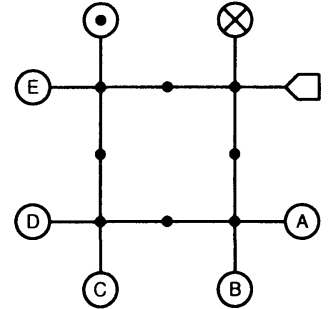

pattern 2

$\begin{array}{ll}\square \text { AGV's pool node } & \times \text { machine node } \\ \bigotimes \text { input node } & \bullet \text { other node } \\ \bigotimes \text { output node } & - \text { guide link }\end{array}$

Figure 2 Examples of Guide Network

\subsection{Comparison of Scheduling by GGC and by the Usual Ways}

Our method on the courses of choosing a job to carry explained in 2.2 is compared here to the conventional ways. 


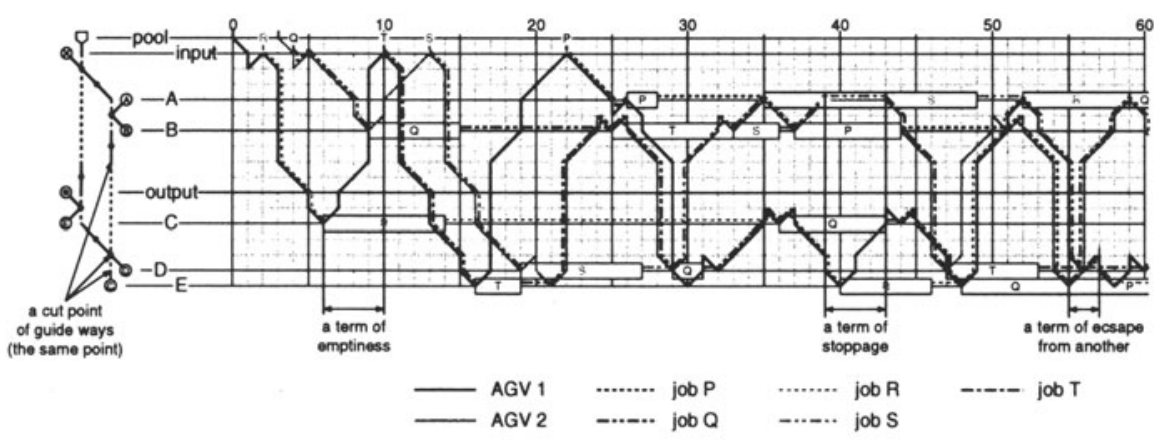

Figure 3 Reduced GGC Used in This Paper

By definition, GGC has 1 more dimensions than that of its map of the plant. Therefore, the charts for the examples of patterns mentioned above should be solid figures. The charts made here, however, are reduced to plain ones by cutting and expanding guide links for the problem on expression. An instance of the reduced chart for the example of loop pattern is in Figure 3. The cut points of the guide ways are here linked with a broken line. Since thier ends are on the identical point, a locus on it is vertical to the time axes.

The more significant course requires information on extra time in AGV operation, i. e., terms of emptiness of the vehicle, of stoppage and of escape from another. GGC gives this information by the way shown in Figure 3. It should be noted that terms in which each vehicle is at the pool before and after its schedule are regarded as terms of stoppage.

On the other hand, the process to be compared is as follows:

- Make Gantt chart in latest-assignment.

- Assume that any job which finished each task on a machine calls all the vehicles.

- Plan the path from the node where a vehicle has finished its previous plan to where a job is calling it and then to the destination of the job. Choose the job whose work time on next machine will start earliest if plural jobs are calling it.

- Choose the vehicle which will arrive earliest where the job is waiting if plural vehicles respond a plan to it. Abandon the plan if another vehicle on its plan is possible to arrive there even earlier.

- Give the way of the vehicle whose load will start its next work time later so that the both plans are expanded as little as possible if one plan crosses another chosen plan.

These rules are selected out of some other variations on results of preparatory schedulings under various rules of them. 
Table 2 Results of Scheduling by Two Methods

\begin{tabular}{lrrr} 
request No. & 1 & 2 & 3 \\
\hline total time of pattern 1 & & & \\
$\quad$ method using GGC & 95 & 96 & 93 \\
conventional way & 107 & 110 & 112 \\
\hline total time of pattern 2 & & & \\
$\quad$ method using GGC & 93 & 99 & 102 \\
$\quad$ conventional way & 112 & 116 & 117 \\
\hline the number of optimum & 3 & 1 & 1 \\
\hline
\end{tabular}

The results of applying the two methods to the requests in Table 1 on the patterns in Figure 2 are compared in Table 2. The total time are defined here: the time from the point when the first vehicle leaves the pool node to the point when the last job arrives at the output node on condition that then all the vehicles can return to the pool. In job-shop requests which have more than one optimal schedule, the best total time is reported as well as the number of the optimums.

Table 2 shows that the total time obtained with our method is shorter than that obtained with the conventional way though the orders of the tasks on each machine are not optimum. This means that our method is superior to the conventional ways even if one of the optimums is found in the conventional way. The numbers reported as using GGC are achieved in only 2 repetitions of processes with a few back-tracking. The solutions for the request 1 are illustrated in Figure 4 and 5.

\subsection{Consideration on Effectiveness of GGC}

The results above is in favor of the proposition that scheduling by GGC is effective. It can be argued that GGC and the course to take carriage efficiency as an index of plans perform effectively on condition that AGV operation is a significant factor of scheduling problem.

The purpose of GGC is to express constraints due to AGV on scheduling space. So it is trivial that GGC brings little effect in such cases that AGV operation is hardly influential on the whole schedule. In fact the examples taken here are prepared so that one moving time is not so less than one work time and so that the number of vehicles is just enough for the scheduling.

On this condition, the idle time of each job, i. e., the time neither at work nor under ideal carriage, which decides the total time is subject to the extra 

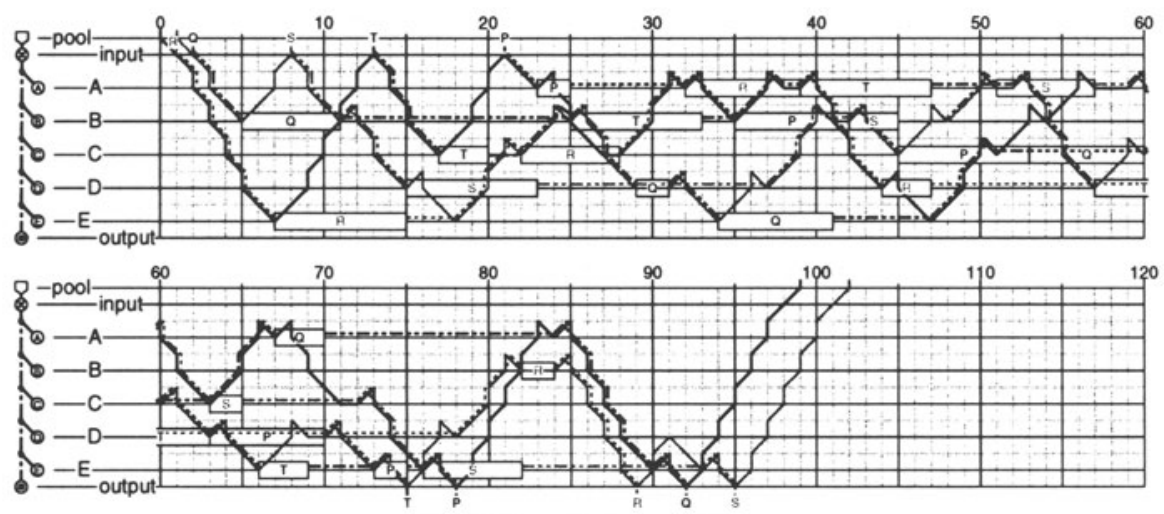

(a) with the method using GGC

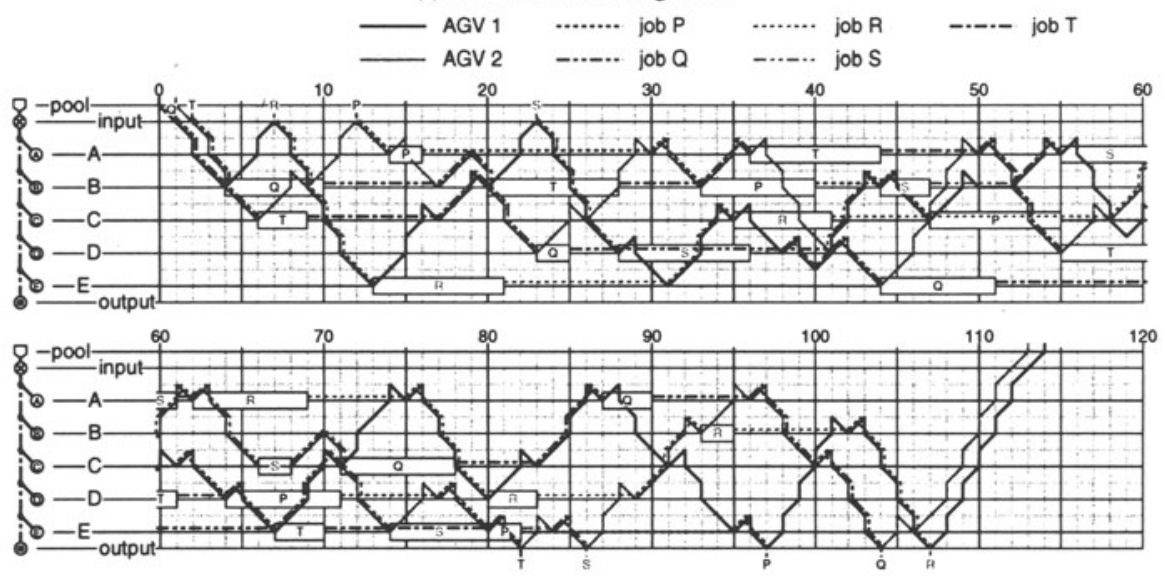

(b) with the conventional way

Figure 4 Solutions for Job-Shop Problem with AGV (request 1, pattern 1)

time of the vehicles. Time of stoppage and of emptiness of a vehicle is equivalent to the idle time of the jobs which wait for it. Time of escape from another vehicle is the idle time of what it is carrying. It is thought that GGC is so effective because it facilitates to sum up the extra time.

Another reason for the effectiveness of GGC is considered to be the various and relatively global informations that human scheduler is able to grasp. He can see at a glance not only carriage efficiency already given but also, for instance, differences between finishing times, positions of jobs, possible places for vehicles to stay away from collision and so on. Such information aids the scheduler to balance the carriage efficiencies between vehicles at local schedules and through the period of the whole schedule and to confirm that. 

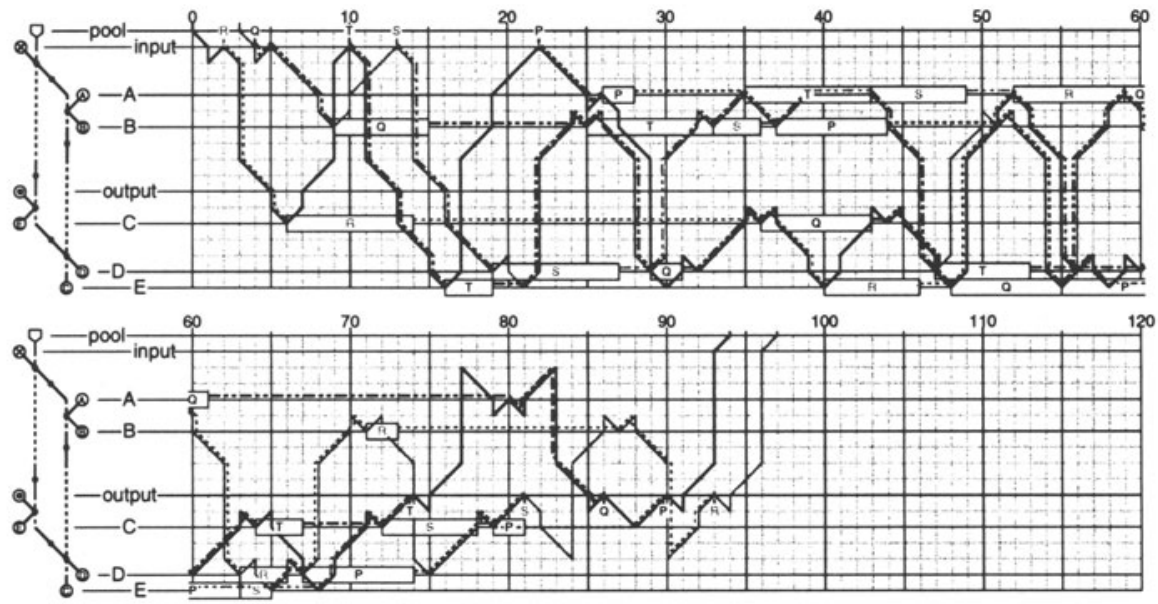

(a) with the method using GGC
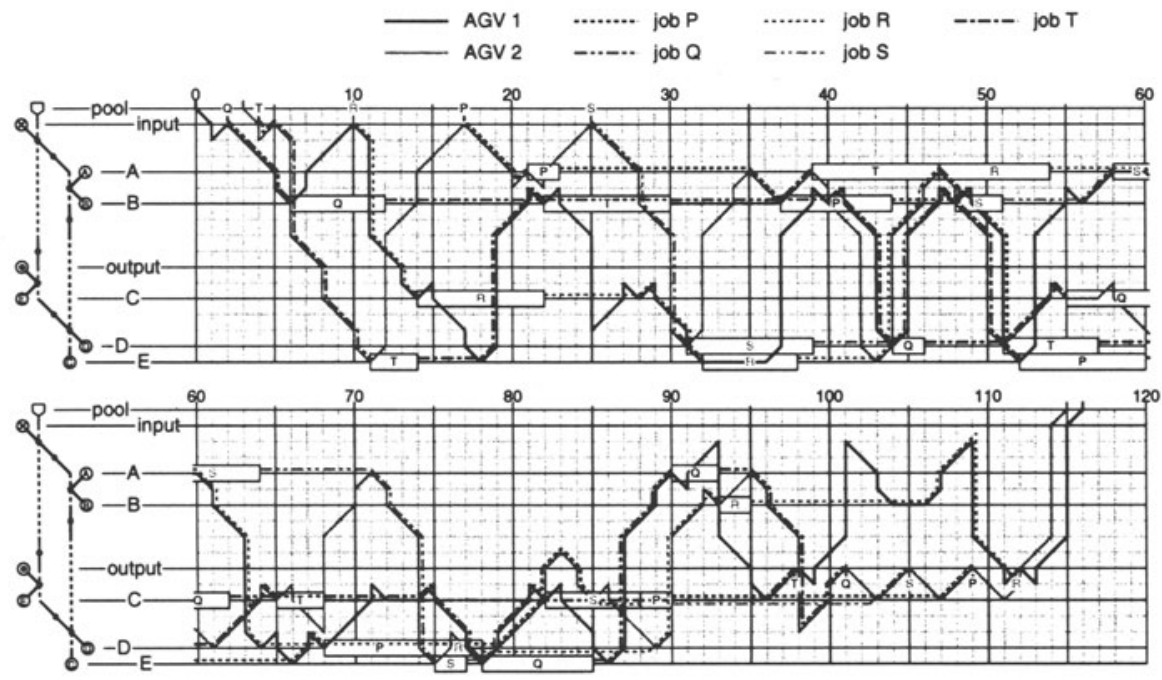

(b) with the conventional way

Figure 5 Solutions for Job-Shop Problem with AGV (request 1, pattern 2)

\section{CONCLUSION}

It has been shown that GGC is effective on manual scheduling for production systems with AGV. To make carriage efficiency of vehicles as high as possible brings an excellent solution for job-shop problem on condition that constraints due to AGV system are dominant. GGC visually supplies indexes of the carriage efficiency and other various global information. The scheduling method 
with it is more effective than the conventional ways based on the calls from jobs which have finished there tasks on machines. Furthermore, it is affirmed that the superiority in the effectiveness of our method using GGC is kept the same even if an optimal order of the tasks on each machine is found.

\section{REFERENCES}

Adelsberger, H. H. and Conen, W. (1995) Scheduling Utilizing Marker Models, in Computer Integrated Manufacturing: Proceedings of the 3rd International Conference (ed. J. Winsor, et al. (Ed.)), 1, 695-702, World Scientific, Singapore.

Błazewicz, J., et al. (1993). Scheduling in Computer and Manufacturing Systems, Springer-Verlag, Berlin.

Kurbel, K. and Ruppel, A. (1995) Integrating Intelligent Job-Scheduling into a Real-World Production Scheduling System, in Computer Integrated Manufacturing: Proceedings of the 3rd International Conference (ed. J. Winsor, et al.), 1, 679-86, World Scientific, Singapore.

Kwok, A. D. and Norrie, D. H. (1992). An Intelligent Agent System for Automated Guided Vehicle Control, in Proceedings of the International Conference on Object Oriented Manufacturing Systems (ed. D. H. Norrie, et al.), 263-70, Univ. of Calgary.

Okino, N. (1995) Theory of Biological Metaphor and its Application to Reactive Scheduling, in Conference Proceedings: IFIP WG5.7 Working Conference on Managing Concurrent Manufacturing to Improve Industrial Performance, September 1995 (ed. R. L. Storch), 504-13. Univ. of Washington.

\section{BIOGRAPHY}

Hideaki Hase recieved M. S. degree in engineering from the Division of Applyed Systems Science, Kyoto University in 1994. He was with Kyoto University from 1994 to 1996 as a reserch associate. Since 1996 he has been with The University of Shiga Prefecture where he is a research associate. His research interests center on procedual programming language.

Norio Okino was born in Tokushima prefecture in Japan, 1933. After graduating Doctor cource of Kyoto University, 1961, he started his research life as associate professor from 1968 to 1987 and worked on CAD/CAM, Intelligent Manufacturing Systems, Bionic Manufacturing Systems, etc. He returned to Kyoto University on 1987 and left his job on reaching retiring age, 1997. At this morment, he is a professor of The University of Shiga Prefecture and professor emeritus of Kyoto University and Hokkaido University. 